\title{
FRANCISCO LAPORTA, LIBORIO HIERRO Y LA VIGENCIA UNIVERSAL DE LOS DERECHOS HUMANOS
}

\author{
Macario Alemany \\ Universidad de Alicante
}

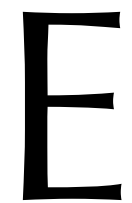

n mi aportación al homenaje a los profesores Francisco LAPORTA y Liborio HIERRO voy a comentar brevemente un aspecto de su posición sobre la vigencia universal de los derechos humanos: me referiré a la cuestión del alcance de los derechos humanos desde el punto de vista de los obligados y, en particular, a la necesidad de afirmar la existencia de deberes positivos generales de ayuda mutua para realizar los derechos de igualdad. A mi juicio, con algunas diferencias de matiz, la posición de ambos autores es similar y puede ser tratada conjuntamente.

Francisco LAPORTA, en su artículo «Sobre el concepto de derechos humanos», nos propone construir una definición por género y diferencia específica. El género, la noción de derecho subjetivo tendría, a su juicio, los siguientes componentes: «a) La adscripción a todos y cada uno de los miembros individuales de una clase de... b) una posición, situación, aspecto, estado de cosas, etc. c) que se considera por el sistema normativo un bien tal que constituye una razón fuerte... d) para articular una protección normativa en su favor a través de la imposición de deberes u obligaciones, la atribución de poderes e inmunidades, la puesta a disposición de técnicas reclamatorias, etc.» (LAPORTA, 1987: 31). La diferencia específica, la noción de «derechos humanos», vendría establecida por las propiedades de universalidad, inalienabilidad y carácter absoluto.

Con respecto a la universalidad, LAPORTA nos recuerda que la universalidad predicada de los derechos humanos no puede ser la «lógico-formal» puesto que sería irrelevante, sino que debe ser la universalidad material o de contenido, si bien se toma en consideración una única propiedad: la de ser «ser humano». Si ninguna circunstancia o condición de un «ser humano» puede ser relevante para negarle un derecho humano, entonces hay que situar fuera del ámbito del sistema jurídico positivo a estos derechos puesto que ningún sistema es universal en este sentido y — dice LAPORTA— - «por lo que a mí respecta tal empresa parece imposible» (LAPORTA, 1987:33). Por ello, nuestro autor opta por situarlos en el ámbito de la moralidad. Ahora bien, la teoría ética ha vinculado tradicionalmente el surgimiento de los derechos a situaciones institucionales (por ejemplo, las promesas) o posicionales (los derechos y obligaciones de rol), de modo que la introducción de la noción de derechos humanos universales supone una «mutación», una «superación» paulatina de una moralidad «local» en favor de una universal. LAPORTA destaca dos implicaciones de esta transformación: primera, que el diseño de 
los derechos debe ser de una gran abstracción, «porque un catálogo excesivamente minucioso y concreto corre el peligro inmediato de ser inaplicado en determinados contextos culturales» (LAPORTA, 1987: 34); y, segunda, que la universalidad se proyecta también sobre los obligados, de manera que se fundamentan obligaciones generales y, también, deberes positivos generales. La «decisiva mutación» de la ética reside precisamente en el cambio de interpretación del principio de solidaridad: de un principio de «caridad» a uno de «exigencia ética fuerte»; de una ética de la beneficencia a una ética de la justicia. En este nuevo marco moral, en el que todos estamos obligados a actuar en defensa de los derechos de todos, LAPORTA se pregunta: «¿Estamos seguros de que puede, entonces, seguir ampliándose ilimitadamente el catálogo de derechos humanos? ¿Estamos seguros de que puede seguir postulándose su fuerza especial?» (LAPORTA, 1987: 36).

Liborio HiERRO, en su artículo «Justicia global y justicia legal. ¿’Tenemos derecho a un mundo justo?», defiende una vinculación conceptual entre la exigencia de justicia global y la universalidad de los derechos humanos. A su juicio, esta vinculación permite interpretar el art. 28 de la Declaración Universal de Derechos Humanos ${ }^{1}$ como un derecho moral de todos a un orden global justo. La teoría de la justicia cosmopolita estaría, de este modo, fundamentada en la teoría de los derechos humanos como derechos universales y en una plausible interpretación del mencionado art. 28.

HIERRO evalúa este punto de vista en relación con lo que considera los tres principales problemas para la justicia global: la guerra, el hambre y la extrema pobreza y, en tercer lugar, la degradación medioambiental. En relación con los mismos, habría tres respuestas posibles: 1) de acuerdo con la tradicional concepción hobbesiana de las relaciones internacionales, las comunidades políticas están en una suerte de estado de naturaleza y no se deben nada moralmente entre sí; la solidaridad es un acto siempre supererogatorio; 2) se pueden dar obligaciones de respeto y ayuda mutua entre los Estados pero, a falta de una autoridad política superior, dichas obligaciones tienen carácter moral y no jurídico; como mucho, pueden juridificarse como consecuencia de la propia autonomía de los Estados para vincularse por medio de acuerdos voluntarios, y 3) «todas las personas tienen un derecho a vivir en paz y/o un derecho a disfrutar de los medios necesarios para subsistir y/o un derecho a la conservación del medio ambiente» (HIERRO, 351); se trata de «derechos universales», lo cual implica «que su contenido es un bien que es de todas y cada una de las personas, es decir que forma parte de lo que es suyo y que, por ello, la justicia —entendida como dar a cada uno lo suyo- exige satisfacer estos derechos» (ibid.). Esta tercera respuesta defendida por HIERRO, asume, al igual que LAPORTA, que los derechos humanos, universales y absolutos, no solo demandan el cumplimiento de obligaciones negativas sino también de obligaciones positivas.

Tanto para HIERRO como para LAPORTA, las obligaciones generales positivas, vinculadas de forma especial al derecho a la igualdad, plantean un problema especial. Las obligaciones generales negativas podrían cumplirse con una coordinación mínima. En este sentido, HIERRO, que aboga por un orden mundial federal, poliárquico, descentra-

1 «Toda persona tiene derecho a que se establezca un orden social e internacional en el que los derechos y libertades proclamados en esta Declaración se hagan plenamente efectivos». 
lizado y democrático, señala que si todas las Constituciones de los Estados, en el marco de ese orden legal mundial, reconocieran el derecho constitucional a la seguridad y a la libertad de sus ciudadanos, entonces dicho derecho habría obtenido una efectividad universal. Por el contrario, la vigencia de una igualdad universal parece requerir de una coordinación fuerte que solo cabe alcanzar en el contexto de instituciones globales.

A este respecto, LAPORTA considera que el cumplimiento de los deberes positivos generales de ayuda mutua, más allá de los ejemplos sencillos como el del rescate del niño que se ahoga en el estanque, solo es exigible, cuando gravitan sobre todo un colectivo, como deberes positivos y especiales de ayuda en el seno del colectivo (LAPORTA, 1986). Para LAPORTA, las exigencias morales derivadas de la universalidad de los derechos y, en particular, del derecho a la igualdad únicamente son realizables en el contexto de las instituciones, llamadas a transformar estas demandas morales en un orden legal que debe ser, además, global. Pero nuestro autor, como hemos visto, no cree posible generar un orden legal global; desde luego, no le parece posible tal cosa, siempre que se siga tomando como ideal para tal orden global el ideal jurídico del imperio de la ley.

En este punto de las obligaciones de ayuda, HIERRO coincide plenamente con LAPORTA, a quien recurre para afirmar que «lo que estas demandas suponen, en términos morales, es que necesitamos una organización política para "posibilitar el cumplimiento" de un deber positivo general de ayuda, un deber que resulta la condición necesaria para que el ideal de igualdad rompa su estrecha y discriminadora construcción constitucional estatal» (HIERRO, 368). Para HIERRO, la cuestión de la posibilidad de tal orden global es difícil y cita a KELSEN para señalar que si bien el proyecto de un Estado mundial es «punto menos que un proyecto utópico» cabe pensar en su realización «después de un largo y lento desarrollo, sobre todo si se fomenta ese desarrollo por medio de una labor política consciente en el campo ideológico» (KELSEN, 1974: 50 y 175).

En resumen, nuestros autores coinciden en afirmar que la universalidad de los derechos humanos implica obligaciones morales generales que, cuando se trata de derechos de igualdad, son de carácter positivo (obligaciones de ayuda y no solo de abstenerse de dañar), pero que tales obligaciones solo pueden ser cumplidas en el contexto de instituciones que las transforman en deberes positivos especiales de cada individuo. Para LAPORTA, además, la universalidad de los derechos humanos aconseja una lista corta de derechos, puesto que cada ampliación hace más difícil su realización. HIERRO, finalmente, en el estado actual de las cosas, considera prioritario afirmar la existencia de un deber moral básico de todos a que exista una institución político-jurídica con poder coercitivo que asegure un orden legal en el que los otros derechos sean realizables, un derecho a un mundo justo.

A partir de aquí quisiera plantear un interrogante que me suscita la posición de estos autores. Me pregunto si su posición sobre la posibilidad de vigencia universal de los derechos humanos no pone de manifiesto una valoración exagerada del principio del imperio de la ley, al que, se diría, se le termina otorgando una prioridad lexicográfica sobre otros valores como, por ejemplo, el valor de la igualdad. En primer lugar, cabe preguntarse por el alcance de la transformación radical de la ética que, como señala LAPORTA, supone el universalismo de los derechos humanos. Ambos autores sostienen que la medida de las obligaciones que entraña dicho universalismo (obligaciones de 
todos para con todos) solo la pueden concretar instituciones: por ejemplo, mediante un sistema impositivo redistributivo o, en el orden global, mediante un tribunal penal internacional, etc. Pero se diría que dicha transformación radical afecta también a la idea misma de las instituciones y, en particular, la cuestión que quiero plantear es si la afirmación de una ética universalista no debería producir una transformación misma en la idea de Derecho.

HIERRO se hace eco, en el artículo que vengo comentando, de la propuesta de BulL de adoptar un modelo neo-medieval para orientar la extraordinaria complejidad de un orden legal universal. La calificación de medieval sigue teniendo una fuerte emotividad desfavorable, pero lo cierto es que hay una evidente base para la analogía. Desde el punto de vista historiográfico, la idea clave para entender el tránsito del Medioevo a la Edad Moderna es la del surgimiento de la soberanía estatal, del mismo modo que la idea clave para interpretar la realidad político-jurídica de nuestro tiempo es la de la crisis de la soberanía estatal; la simetría es evidente. Esta crisis se debe en gran medida, como sabemos, al fenómeno de la globalización: CAPELla, FerRAJOli, Atienza o el propio LAPORTA han descrito con lucidez el impacto de la misma. Pero no hay que olvidar que la pérdida de la soberanía estatal está lógicamente presupuesta en la afirmación de la prioridad de los derechos humanos. De algún modo, la crisis del derecho estatal y de la comprensión del mismo en términos positivistas (el derecho con cualquier contenido de KELSEN) comienza al término de la Segunda Guerra Mundial, cuando arranca significativamente, como han señalado PERELMAN o ATIENZA, una nueva concepción, argumentativa, del Derecho. En mi opinión, algunos fenómenos actuales como el diálogo entre Cortes estatales que ejercen control judicial de constitucionalidad para interpretar sus respectivas constituciones, la proliferación del denominado «soft-law», la importancia creciente de los principios en el Derecho o, en general, la extensión de la ideología del constitucionalismo y la crisis del positivismo, son, entre otras cosas, manifestaciones de la pretensión de vigencia universal de los derechos humanos.

En uno de sus ensayos sobre HobBes, sostiene BobBio que la idea dominante de la teoría política de HOBBES es la de la conservación de la unidad frente a la anarquía: «el mal al que más teme - dice BOBBiO— y contra el cual se siente llamado a erigir la suprema e insuperable defensa de su sistema filosófico, no es la opresión que deriva del exceso de poder, sino la inseguridad, que por el contrario deriva del defecto de poder» (BoвBIO, 53). Esta tesis de BOBBIO se apoya en las circunstancias históricas de la vida de HobBES y en que su sistema filosófico no puede ser aceptado si no se asume una jerarquía de valores de acuerdo con la cual es peor la anarquía, por defecto de poder, a la opresión, por exceso. A mi juicio, nuestros dos autores comparten «un mal al que más temen» y este no es otro que el de la sustitución del poder de las legislaturas democráticas por el de jueces o funcionarios, legitimados no por la voluntad popular sino por la pretendida racionalidad de sus decisiones. Frente al cuestionamiento de las estructuras político-jurídicas de la modernidad, su respuesta es la reafirmación del valor del imperio de la ley como condición necesaria de la justicia, a pesar de que ello suponga admitir la imposibilidad de su realización universal. HIERRO es, a mi juicio, más optimista que LAPORTA sobre la posibilidad de un orden legal mundial y parece más abierto a considerar configuraciones complejas del poder compatibles con un ideal democrático del imperio de la ley, también se diría que es más proclive a creer 
en una «punto menos que utópica» realización universal de los derechos de igualdad. Sin embargo, se trata de una cuestión de énfasis y ambos autores coinciden en una misma concepción del Derecho fundamentalmente positivista que parece haber sido desbordada por la historia.

No estoy defendiendo un punto de vista ingenuo sobre la globalización tal y como se está desarrollando. Tampoco quiero despreciar el miedo a un activismo irresponsable de los derechos humanos, que colabora inadvertidamente con las ideologías «liberticidas» en destruir las condiciones de posibilidad de los mismos (por ejemplo, despreciando la importancia de la seguridad jurídica). Creo que hay muchas razones para el pesimismo. De hecho, resulta paradójico pensar en el problema de la extensión de los ideales igualitarios de la socialdemocracia más allá de los límites de las fronteras estatales, al tiempo que no se sabe poner freno a la destrucción de ese mismo ideal también dentro de los Estados. La ideología neoliberal parece extenderse ya casi sin oposición por todos lados. Pero, en conclusión, me parece que también por razones morales hay que, utilizando una expresión de ATIENZA y RUIZ MANERO, dejar atrás el positivismo jurídico; si se quiere, se trataría de afirmar un postpositivismo ético.

No quisiera terminar estas líneas sin mostrar mi agradecimiento personal a LAPORTA y HIERRO. Ambos son miembros de una generación que ha contribuido significativamente a cuatro décadas consecutivas de progreso en nuestro país, a partir de una exitosa transición política. No creo que sea exagerado decir que jamás hubo en España una situación de respeto por los derechos de tal amplitud, por el número de beneficiados, ni tan duradera. Igualmente, han sido protagonistas de una modernización radical de la Universidad pública y, en particular, de la filosofía del Derecho española, que ahora mismo está reconocida mundialmente como de primer nivel. Personalmente me he beneficiado de su magisterio a través de sus escritos y participación frecuente en las actividades académicas en el departamento de filosofía del Derecho de la Universidad de Alicante. Liborio HIERRO ha estado muy presente, además, en mi carrera académica participando en los tribunales de mi tesis de licenciatura y, posteriormente, de mi tesis doctoral. Ahora, cuando parece que los tiempos están cambiando para peor, cuando nos encontramos ante unas amenazas cada vez más serias a la cultura de los derechos, como son el populismo, el nacionalismo, las nuevas formas de autoritarismo, la crisis del cuarto poder, etc., cuando también se invierte la tendencia a la mejora en la Universidad pública, me parece que el papel más digno que puede desempeñar mi generación es el de tratar de preservar lo máximo del legado de la que nos antecede y en la que Liborio HIERRO y Francisco LAPORTA han destacado especialmente.

\section{BIBLIOGRAFÍA}

AtienZA, M., 1991: Las razones del Derecho. Teorías de la argumentación jurídica, Madrid: Centro de Estudios Constitucionales.

- 2013: «Constitucionalismo, globalización y Derecho», en Podemos hacer más. Otra forma de pensar el Derecho, Madrid: Pasos perdidos.

BobBIo, N., 1991: Thomas Hobbes, Barcelona: Paradigma.

Bull, H., 2005: La sociedad anárquica. Un estudio sobre el orden en la política mundial, Madrid: Los libros de la catarata. 
CAPELLA, J. R., 2005: La globalización: ante una encrucijada político-jurídica, en M. EsCAMILLA y M. SAAVEDRA (eds.), Law and Justice in a Global Society, Granada: Anales de la Cátedra Francisco Suárez.

FERRAJOLI, L., 2005: La crisis de la democracia en la era de la globalización, en M. EsCAMILLA y M. SAAvedRA (eds.), Law and Justice in a Global Society, Granada: Anales de la Cátedra Francisco Suárez.

Hierro, L. L., 2009: «Justicia global y justicia legal. ¿Tenemos derecho a un mundo justo?», Doxa. Cuadernos de Filosofía del Derecho, vol. 32: 341-374.

Kelsen, H., 1974: Derecho y paz en las relaciones internacionales, México: Editora Nacional.

LAPORTA, F., 1986. «Algunos problemas de los deberes positivos generales. Observaciones a un artículo de Ernesto Garzón Valdés», Doxa. Cuadernos de Filosofía del Derecho, vol. 3: 55-63.

- 1987: «Sobre el concepto de derechos humanos», Doxa. Cuadernos de Filosofía del Derecho, vol. 4: 23-46.

- 2005: «Globalización e imperio de la ley. Un texto provisional para el debate con algunas dudas y perplejidades de un viejo westfaliano», en M. EsCAmiLla y M. SAAVEDRA (eds.), Law and Justice in a Global Society, Granada: Anales de la Cátedra Francisco Suárez.

Perelman, Ch., 1979: La lógica jurídica y la nueva retórica, Madrid: Civitas. 